\title{
Influence of the local gas injection on the flow structure in the boundary layer
}

\author{
Evgeny Maslov ${ }^{1,2, *}$, Nadezhda Savkina ${ }^{1}$, Irina Zharova $^{1}$, Valery Faraponov ${ }^{1}$, Evgeny \\ Kozlov ${ }^{1}$, and Vladislav Matskevich ${ }^{1}$ \\ ${ }^{1}$ Tomsk State University, 634050 Tomsk, Russia \\ ${ }^{2}$ National Research Tomsk Polytechnic University, 634050 Tomsk, Russia
}

\begin{abstract}
The results of an experimental and numerical study of the interaction of supersonic flow around an axisymmetric model, $\mathrm{M}=(2 \div 5)$, with a jet of gas injected locally into the boundary layer are presented.
\end{abstract}

\section{Introduction}

The maneuverability of the supersonic aircraft on the flight trajectory can be realized by means of local gas injection from the surface into the boundary layer. Thus, at the drawingboard stage it becomes necessary to solve the problem of determining the main parameters and flow structure in the boundary layer depending on the blowing conditions.

The aim of this paper is an experimental-theoretical study of the influence of the local gas injection into a boundary layer under the flow around an axisymmetric model in the range of Mach numbers $\mathrm{M}=(2 \div 5)$.

\section{Methodology and results of experimental studies}

Experiments to determine the drag coefficient when supersonic air flow around an axisymmetric body were carried out on a pulsed aerodynamic installation [1]. The range of realizable flow modes: the number $\mathrm{M}=(2 \div 5)$; the stagnation pressure at the diffuser nozzle section forming the supersonic flow, $P_{0}=(0.15 \div 0.3) \mathrm{MPa}$ at static pressure $P=(0.03 \div 0.07) \mathrm{MPa}$; the stagnation temperature of the incoming air is $T_{0}=(17.5 \div 250){ }^{\circ} \mathrm{C}$. The durability of the pulsed aerodynamic installation, depending on the test conditions, is $(1.0 \div 3.0) \mathrm{s}$.

The models in the form of a cone with a half angle of $15^{\circ}$ were subjected to tests on the aerodynamic installation (Fig. 1, a). The blowing of gas into the boundary layer was carried out through sampling holes. The general view of the model is shown in Fig. 1, b. In Fig. 1, $\mathrm{c}$ the photo of the model installed in the test section of the aerodynamic installation is presented.

\footnotetext{
* Corresponding author: maslov eugene@mail.ru
} 


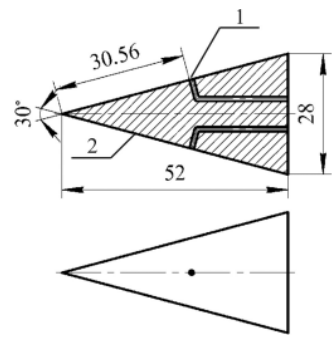

a

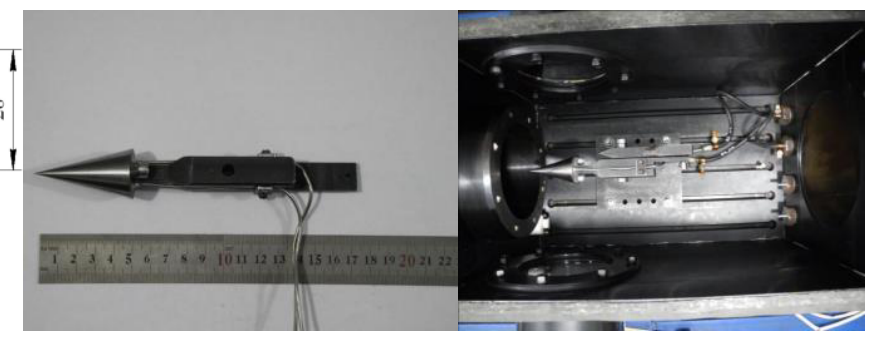

b

$\mathrm{c}$

Fig. 1. Scheme (a), general view (b) and photo of the model installed in the test section of the aerodynamic installation (in): 1 - sampling hole; 2 - the surface of the model.

To determine the value of the drag coefficient in experiments using the tensometric balance, the force acting on the surface of the model when supersonic air flow around model was continuously measured in the range of Mach numbers $M=(2 \div 5)$. As a controltest in experimental studies, the results of tests for flow around a cone without injection in the considered range of Mach numbers were used. Fig. 2 shows the results of measurements and the data presented in $[3,4]$. Comparison of the obtained results with the data of other authors showed satisfactory agreement - the maximum difference was $<15 \%$.

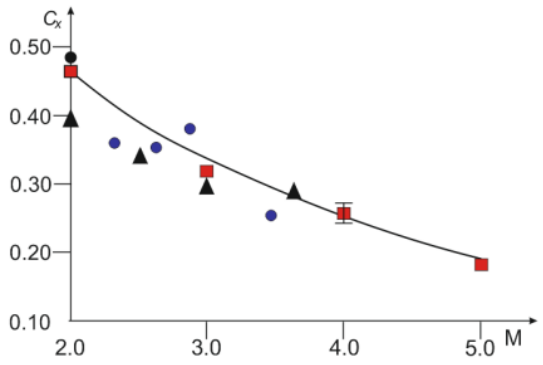

Fig. 2. Drag coefficient of a cone: $\mathbf{-}$ - results of experiments; $\bullet$ - data [3]; $\mathbf{\Delta}$ - data [4].

In table. 1 the value of the drag coefficient of the cone averaged over the series of experiments results at $M=3$ is presented.

Table 1. The drag coefficient, $M=3$.

\begin{tabular}{|c|c|c|c|c|}
\hline \multirow{2}{*}{ Mach number } & $C_{x}$ & $\bar{C}_{x}$ & $\Delta C_{x}$ & $\delta, \%$ \\
\hline \multirow{3}{*}{3} & 0.326 & \multirow{2}{*}{0.324} & 0.006 & \multirow{2}{*}{1.85} \\
\cline { 2 - 2 } & 0.325 & & \multicolumn{3}{|c|}{$\delta_{\text {mean }}=3.64 \%$} \\
\cline { 2 - 3 } & 0.322 & \multicolumn{4}{|c}{} \\
\hline
\end{tabular}

The structure and dynamics of the flow, the characteristic features of the boundary layer formation and the interaction of flows in the boundary layer in the presence of blowing were obtained by the method of shadow visualization. In Fig. 3 video frames of flow around the cone by an air flow $(\mathrm{M}=3)$ with injection through the hole located at the bottom of the model (Fig. 3, a) and without injection (Fig. 3, b) are demonstrated. 


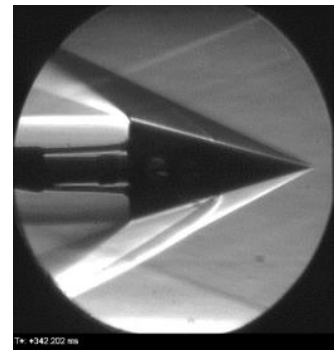

a

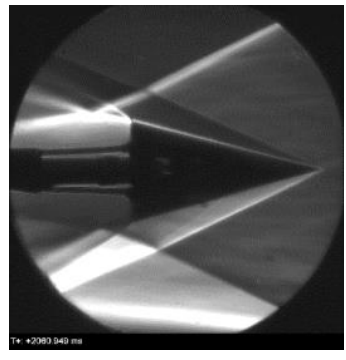

b

Fig. 3. Flow around the cone by an air flow $(M=3)$ with injection (a) and without injection (b).

Comparison of the patterns of flow around the cone without injection and during local blowing through one hole showed a qualitative difference between the interacting supersonic air flow and the surface of the cone. When flowing over a cone with a local blowing through one hole, the angles between the Mach cone and the model surface are different. When a local blowing through one hole, the Mach cone is asymmetric.

\section{Numerical study of supersonic flow around an axisymmetric model under local gas injection}

The temperature and pressure fields for supersonic flow around a cone with allowance for local gas injection into the boundary layer were determined based on numerical simulation of the process. Thermogasdynamic flow characteristics were obtained using the Ansys Fluent software package. The flow of a continuous medium (gas) was modeled by the Navier-Stokes system for turbulent flow of a viscous compressible gas. As a model of turbulence, the SST-model (Menter's model of shear stresses) was used, based on the vortex viscosity hypothesis. Numerical implementation of the solution was carried out on an unstructured grid with different grid resolution (Fig. 4). The grid was clustered near the body surface.

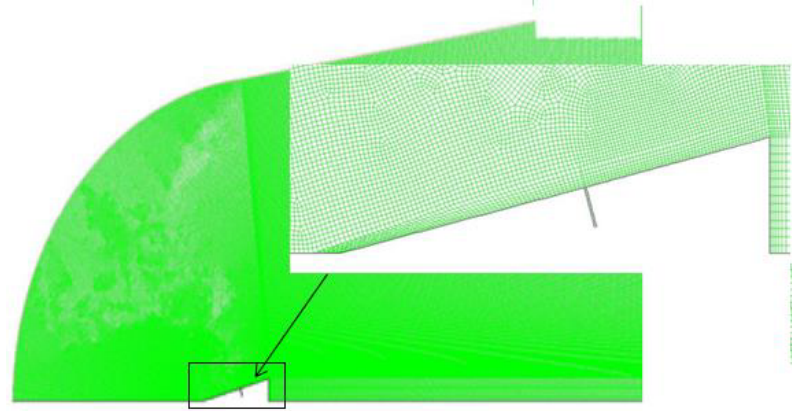

Fig. 4. Grid domain of the problem solution.

The number of grid nodes was chosen from the experimentally determined value of the drag coefficient $C_{x}$.

As an example, in Table. 2 the data of the grid convergence (the number of grid nodes) in the numerical study of the supersonic flow around a cone $(M=3)$ is given, $\delta C_{x}=\frac{\left|C_{x}^{\text {calc }}-\bar{C}_{x}\right|}{\bar{C}_{x}}-$ relative error of the calculated value of the drag coefficient, $\bar{C}_{x}=0.324$ (Table. 2 ). 
Table 2. Grid convergence in supersonic flow around a cone, $\mathrm{M}=3$.

\begin{tabular}{|c|c|c|}
\hline \multicolumn{1}{|c|}{ Cells } & $\boldsymbol{C}_{\boldsymbol{x}}^{\text {calc }}$ & $\boldsymbol{\delta} \boldsymbol{C}_{\boldsymbol{x}}$ \\
\hline 92532 & 0.3189 & 0.0157 \\
\hline 138798 & 0.3212 & 0.0086 \\
\hline 208197 & 0.3239 & 0.0003 \\
\hline 320500 & 0.3241 & 0.0003 \\
\hline
\end{tabular}

On the basis of numerical studies, data of the temperature and pressure distributions when the supersonic flow around the cone were obtained, $M=(2 \div 5)$, with considering the local gas injection into the boundary layer.

In Fig. 5 a graph of the temperature distribution near the cone surface when local blowing is presented, $M=3$, and the angle of attack is $\alpha=0^{\circ}$. An analysis of the obtained results showed the effect of injection on the temperature distribution in the boundary layer. The temperature distribution is essentially nonmonotonic: the highest temperature - from the top of the cone to the sampling hole, a sharp decrease of temperature at the injection segment, a monotonic increase of the temperature from the sampling hole to the base of the cone.

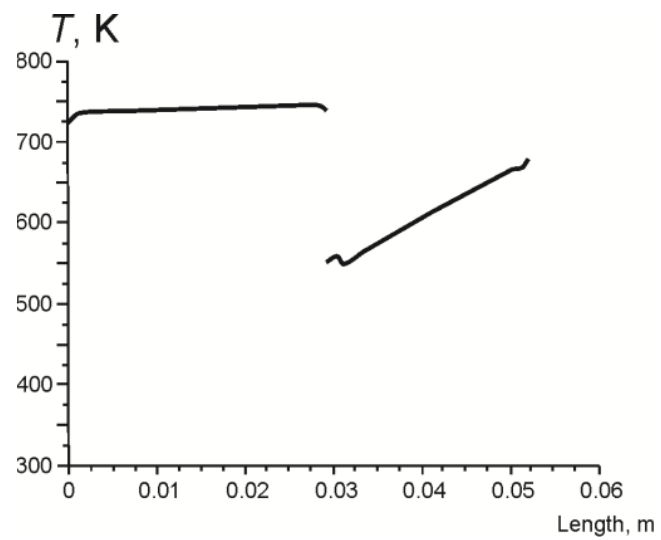

Fig. 5. Temperature distribution near the surface of the cone in the presence of local injection.

Fig. 6 shows the data of the pressure field obtained numerically (Fig. 6, a, c), and the flow structure obtained experimentally (Fig. 6, b). The data presented correspond to the flow around a cone with blowing through two symmetrically located holes at $\mathrm{M}=3$.

The analysis of the experimental and numerical results showed their conformity with the main features of the flow structure in the considered conditions: the formation of the Mach cone, the dynamic perturbation of the flow in the field of injection, the formation of detached flow from the surface at the base of the cone.

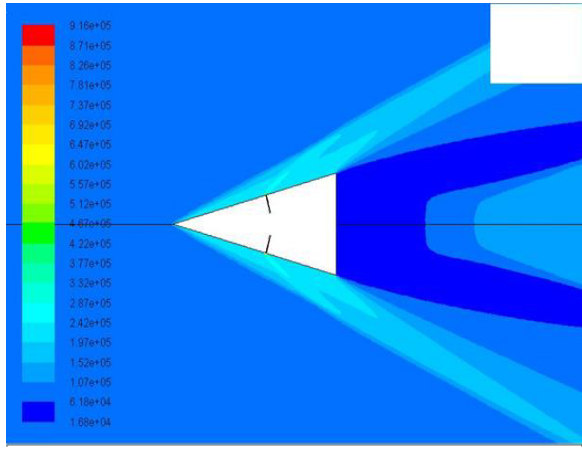

a

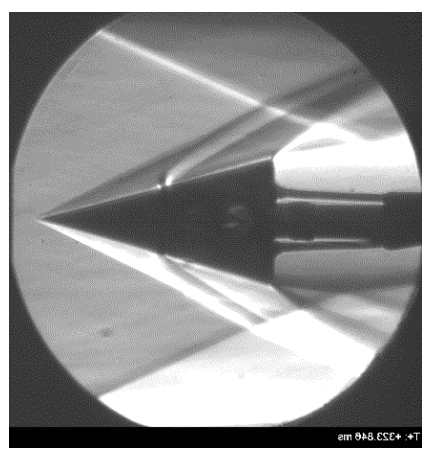

$\mathrm{b}$ 


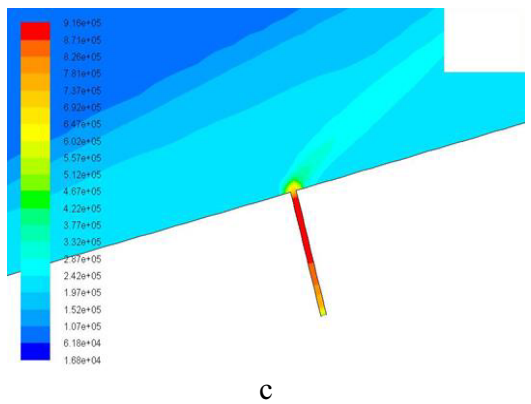

Fig. 6. The pressure distribution (a, c) and the flow structure (b) for flow around a cone with blowing through two symmetrically located holes, $\mathrm{M}=3$.

\section{Conclusions}

Based on the results of an experimental-theoretical study of the effect of local gas injection into the boundary layer when the flow around an axisymmetric model in the range of Mach numbers $\mathrm{M}=(2 \div 5)$, the main thermogasdynamic flow characteristics are determined.

A qualitative analysis of the results obtained when flow around a cone with and without injection showed a significant influence of the injection on the dynamics of the boundary layer and the Mach cone formation.

This research carried out in 2017 was supported by «The Tomsk State University Academic D.I. Mendeleev Fund Program», grant N 8.2.05.2017.

\section{References}

1. E.A. Maslov, V.V. Klochikhin, I.K. Zharova, MATEC Web Conf. 23, 01026 (2015)

2. E.A. Maslov, V.V. Faraponov, N.N. Zolotorev, A.V. Chupashev, V.V. Matskevich, S.Yu. Chizhov, MATEC Web Conf. 92, 01056 (2017)

3. V.G. Artonkin, P.G. Leutin, K.P. Petrov, Scientific works CAI, 92, 1413 (1972)

4. Yu.V. Sheludko, Physical and gas-dynamic ballistic studies (Science, Leningrad, 1980) [in Russian] 